\title{
hsa-microRNA-411-5p regulates proliferation, migration and invasion by targeting the hyaluronan mediated motility receptor in ovarian cancer
}

\author{
FANG HE ${ }^{1}$, DONGYU ZU ${ }^{2}$, CHONG LAN ${ }^{1}$, JUMIN NIU ${ }^{1}$ and XIAOCUI NIE ${ }^{1}$ \\ ${ }^{1}$ Department of Gynecology, Shenyang Women's and Children's Hospital, Shenyang, Liaoning 110011; ${ }^{2}$ The Outpatient \\ Department of the First Garrison, General Hospital of The Northern War Zone, Shenyang, Liaoning 110001, P.R. China
}

Received April 30, 2019; Accepted January 9, 2020

DOI: $10.3892 /$ etm.2020.8899

\begin{abstract}
The mortality rate of ovarian cancer is the highest out of all gynecological malignancies worldwide. Therefore, it is important to understand the mechanisms of ovarian cancer, identify new biomarkers and develop targeted drugs. The role and molecular mechanisms of hsa-microRNA (miR)-411-5p in ovarian cancer have not been fully elucidated. The present study investigated the ovarian cancer cell lines OVCAR-8 and SKOV3. After transfection with miRNA mimics, cell proliferation was monitored by a proliferation assay. Furthermore, cell migration was measured by a cell wound healing assay and cell invasion was measured by Matrigel invasion assays. A miRNA luciferase reporter assay was used to analyze the relationship between miRNAs and the target gene HMMR, which was then further evaluated by gene differential analysis. In the current study, hsa-mir-411-5p was identified as a miRNA regulator of the hyaluronan mediated motility receptor, which negatively regulated the activity of ERK1/2 and ultimately inhibited ovarian cancer cell proliferation and motility. Although hsa-mir-411-5p may have different roles in other types of cancer, the present study suggested that miR-411-5p functions as a negative tumor regulator in ovarian cancer cells, displaying the potential of miR-411-5p as a biomarker for ovarian cancer.
\end{abstract}

\section{Introduction}

Ovarian cancer is the third most common gynecological cancer worldwide, and has the highest mortality rate of all gynecological malignancies $(1,2)$. The 5-year survival rate for patients with early ovarian cancer (stage I or II) is $80-90 \%$, compared

Correspondence to: Dr Xiaocui Nie, Department of Gynecology, Shenyang Women's and Children's Hospital, 87 Danan Street, Shenyang, Liaoning 110011, P.R. China

E-mail: xiaocui_nie@163.com

Key words: ovarian cancer, hsa-microRNA-411-5p, proliferation, invasion, hyaluronan mediated motility receptor with the survival rate of patients with stage III and IV disease, which is only $25 \%(3,4)$. Only $30 \%$ of American patients with ovarian cancer are diagnosed in the early stages in $2016(3,4)$. In patients with recurrent ovarian cancer, treatment is less effective than in patients with a first time diagnosis of ovarian cancer (5). As the number of relapses increases for a particular individual, the disease remission period is reduced (5). At present, there is no effective preventive strategy for ovarian cancer (5). Interventions that reduce the risk of ovarian cancer are particularly important given the limited screening options and high mortality rates, which have not significantly changed over the past several decades (5). Therefore, ovarian cancer is currently a major public health concern. The molecular mechanisms underlying the proliferation, migration and invasion of ovarian cancer cells require further investigation to identify new biomarkers and develop targeted drugs.

MicroRNAs (miRNAs/miRs) are non-coding RNAs $\sim 22$ nucleotides in length, which are involved in cell homeostasis and tumorigenesis (6). The abnormal expression of miRNAs in ovarian cancer is closely associated with the progression and clinical prognosis of the disease $(7,8)$. A number of carcinogenic pathways may influence the same miRNA, and equally, a number of miRNAs may control a post-transcriptional program that affects numerous target genes $(6,7)$. miRNAs are often downstream effectors of tumor relevant protein kinases or mutations, and therefore, targeting miRNAs may be a strategy to increase drug specificity and overcome drug resistance $(6,8)$.

The function and expression of miR-411 has been studied in several types of cancer (9-12). A number of previous studies have reported that miR-411 may function as an oncogene in osteosarcoma and lung and hepatocellular carcinoma (9-12). Contrastingly, a number of studies have reported that miR-411 functions as a tumor suppressor in oral, cervical, breast and colorectal cancer, as well as renal cell carcinoma and glioma (13-21). At present, the role of hsa-mir-411-5p (also known as miR-411-5p) in the development of ovarian cancer is not completely understood. However, it has been reported that the expression of hsa-mir-411-3p in the stable state is low, which is unstable and easy to degrade (22). The present study explored the mechanisms of hsa-mir-411-5p in ovarian cancer cells in vitro. 


\section{Materials and methods}

Cell culture and transfection. The ovarian cancer cell lines OVCAR-8 and SKOV3 were purchased from The Cell Bank of Type Culture Collection of the Chinese Academy of Sciences and American Type Culture Collection, respectively. Cells were maintained in RPMI-1640 medium (Gibco; Thermo Fisher Scientific, Inc.) supplemented with $10 \%$ FBS (Gibco; Thermo Fisher Scientific, Inc.), at $5 \% \mathrm{CO}_{2}$ and $37^{\circ} \mathrm{C} .293$ cells (The Cell Bank of Type Culture Collection of the Chinese Academy of Sciences) were cultured in DMEM (Gibco; Thermo Fisher Scientific, Inc.) supplemented with $4,500 \mathrm{mg} / 1$ glucose and $10 \%$ FBS. The OVCAR- 8 and SKOV3 cells were transfected with $25 \mathrm{nM}$ negative control mimic (MISSION ${ }^{\circledR}$ miRNA, Negative Control 1; cat. no. HMC002; Sigma Aldrich; Merck KGaA) or miR-411-5p mimic (MISSION microRNA Mimic hsa-miR-411-5p; cat. no. HMI0558; Sigma-Aldrich; Merck $\mathrm{KGaA}$ ) at $24 \mathrm{~h}$ after seeding, and cells were seeded into 6-well plates at a density of $1 \times 10^{6}$ cells/well. The sequences used were as follows: Negative control mimic forward, 5'-[AmC6]UGA ACAGUGUUACGUACGAUACC[dT][dT]-3', and reverse 5'-GGUUCGUACGUACACUGUUCA-3'. hsa-miR-411-5p mimic forward 5'-CGUACGCUAUACGGUCUAUCU A[dT][dT]-3', and reverse 5'-UAGUAGACCGUAUAGCGU ACG-3'. Transfection was carried out using Lipofectamine ${ }^{\circledR}$ RNAiMAX Transfection reagent (Thermo Fisher Scientific, Inc.) according to the manufacturer's instructions.

IncuCyte proliferation assay. OVCAR- 8 and SKOV3 cells 2,000 cells/well were seeded into 96-well plates and allowed to adhere at $37^{\circ} \mathrm{C}$ and $5 \% \mathrm{CO}_{2}$. After transfection for $12 \mathrm{~h}$ with miRNA mimics, cell proliferation was monitored by analyzing the occupied area (\% confluence) of cell images at 0,24, 48 and $72 \mathrm{~h}$ time points using the IncuCyte Live Cell Analysis system (Essen BioScience) according to the manufacturer's instructions.

Cell wound healing assay. OVCAR- 8 and SKOV 3 cells $\left(1.5 \times 10^{4} /\right.$ well $)$ were seeded into 96 -well ImageLock plates for $24 \mathrm{~h}$ in RPMI-1640 medium supplemented with $10 \%$ FBS at $37^{\circ} \mathrm{C}$ and $5 \% \mathrm{CO}_{2}$. Following serum starvation (RPMI-1640 medium only) for $12 \mathrm{~h}$ at $37^{\circ} \mathrm{C}$ and $5 \% \mathrm{CO}_{2}$, scratch wounds were made by the IncuCyte ${ }^{\mathrm{TM}}$ Cell Migration kit (Essen BioScience), using the 96-pin wound making tool (WoundMaker). Subsequently, cells were cultured in RPMI-1640 medium supplemented with 4\% FBS for $24 \mathrm{~h}$ at $37^{\circ} \mathrm{C}$ and $5 \% \mathrm{CO}_{2}$. Images were automatically monitored and analyzed every $4 \mathrm{~h}$ for $24 \mathrm{~h}$ by the IncuCyte Live Cell Analysis system (Essen BioScience; magnification, x10). Data were processed and analyzed using IncuCyte ${ }^{\mathrm{TM}}$ Cell Migration Software Application Module (Essen BioScience) for $24 \mathrm{~h}$. Data are presented as the wound width.

Cell invasion assay. OVCAR- 8 and SKOV3 cells $\left(\sim 5 \times 10^{4}-1 \times 10^{5}\right)$ were resuspended in serum-free RPMI-1640 medium and seeded in the top portion of a BioCoat Matrigel Invasion Chamber (Corning, Inc.). The lower compartment of the chamber contained RPMI-1640 medium supplemented with $10 \% \mathrm{FBS}$ as a chemoattractant. After a $28-\mathrm{h}$ incubation at $37^{\circ} \mathrm{C}$ and $5 \% \mathrm{CO}_{2}$, cells on the upper side of the membrane were removed, washed with PBS and fixed in 50\% ethanol for $15 \mathrm{~min}$ at room temperature. Subsequently, cells were stained with Coomassie brilliant blue for $15 \mathrm{~min}$ at room temperature and counted under 10 different microscopic fields using an inverted light microscope (Leica Microsystems; magnification, x100).

$R N A$ isolation and reverse transcription-quantitative PCR $(R T-q P C R)$. To measure miRNA expression, total RNA was isolated from cultured OVCAR-8 and SKOV3 cells using the mirVana ${ }^{\mathrm{TM}}$ miRNA Isolation kit with phenol (Ambion; Thermo Fisher Scientific, Inc.). The mature form of miRNAs were detected using the TaqMan ${ }^{\circledR}$ Advanced miRNA cDNA Synthesis kit with TaqMan Fast Advanced Master mix (Thermo Fisher Scientific, Inc.). The hsa-miR-411-5p TaqMan Advanced miRNA assay kit (assay ID, 478086_mir) and the hsa-miR-26a-5p TaqMan Advanced miRNA assay kit (assay ID, 477995_mir), which was an internal control, were purchased from Thermo Fisher Scientific, Inc. RT-q PCR was performed by Applied Biosystems 7900HT Fast Real-time PCR system (Applied Biosystems; Thermo Fisher Scientific, Inc.). The relative expression was calculated by $2^{-\Delta \Delta \mathrm{Cq}}$ method (23) and expression levels were normalized to hsa-miR-26a-5p.

To investigate the effects of miRNA-411-5p overexpression on the potential miRNA target gene HMMR, total RNA from OVCAR-8 and SKOV3 cells was isolated using the RNeasy Plus Mini kit (Qiagen Sciences, Inc.; cat. no. MD 20874) according to the manufacturer's protocol. RNA was reverse transcribed into cDNA using the High-Capacity cDNA Reverse Transcription kit (Thermo Fisher Scientific, Inc.) according to the manufacturer's protocol. PCR was performed in triplicate using $\mathrm{SYBR}^{\circledR}$ Select Master mix (Thermo Fisher Scientific, Inc.) on a 7900HT Fast Real-time PCR system (Applied Biosystems; Thermo Fisher Scientific, Inc.). The primer sequences used were as follows: HMMR forward, 5'-CCAGGTGCTTATGATGTTAAAACT-3' and reverse, 5'-TGAGATTCCTTCTTTGATTCCGA-3'; and $\beta$-actin forward, 5'-ATTGGCAATGAGCGGTTCCG-3' and reverse, 5'-CGTGGATGCCACAGGACTCC-3'. The following thermocycling conditions were used: $95^{\circ} \mathrm{C}$ for $5 \mathrm{~min}$; 40 cycles of $95^{\circ} \mathrm{C}$ for $10 \mathrm{sec}, 60^{\circ} \mathrm{C}$ for $20 \mathrm{sec}$ and $72^{\circ} \mathrm{C}$ for $20 \mathrm{sec}$. Relative expression was calculated using the $2^{-\Delta \Delta \mathrm{Cq}}$ method (23) and expression levels were normalized to $\beta$-actin.

Bioinformatics analysis and Luciferase reporter assay. starBase (version 2.0; starbase.sysu.edu.cn) online database was used to predict that HMMR was a target of miRNA-411-5p. The human HMMR 3'UTR fragment (337 bp) was PCR-amplified from the genomic DNA and subcloned into the pMIR-REPORT luciferase construct (Thermo Fisher Scientific, Inc.) using the following cloning primers: Forward, 5'-TTGGTCCTACCTATTATCCTTCTA-3'; reverse, 5'-AATGACTTACTGTGTAATTTTATTTC-3'. The mutant HMMR 3'UTR was made using a QuikChange Site-directed Mutagenesis kit (Agilent Technologies, Inc.) and confirmed using Sanger sequencing. 293 cells of $60 \%$ confluence in 24-well plates were co-transfected with the pMIR-REPORT firefly luciferase reporter gene construct, the pRL-TK Renilla luciferase construct (for normalization) and $25 \mathrm{nM}$ miRNA mimics per well using Lipofectamine 2000 reagent (Invitrogen; Thermo Fisher Scientific, Inc.). Cell extracts were 
prepared $48 \mathrm{~h}$ after transfection and the luciferase activity was measured using the Dual-Luciferase Reporter Assay system (Promega Corporation).

Western blotting. Total protein from OVCAR-8 and SKOV3 cells was extracted using RIPA buffer (Beyotime Institute of Biotechnology). Total protein was quantified using a bicinchoninic acid assay kit (Beyotime Institute of Biotechnology). $40 \mu \mathrm{g}$ protein/lane was separated on a $10 \%$ SDS-PAGE gel and then transferred to Hybond membranes (GE Healthcare). Membranes were blocked for $1 \mathrm{~h}$ in 5\% milk in TBST at room temperature. For immunoblotting, membranes were incubated overnight at $4{ }^{\circ} \mathrm{C}$ with antibodies targeted against HMMR (cat. no. ab124729; 1:1,000; Abcam), phosphorylated-Erk1/2 (cat. no. 4377; 1:1,000; Cell Signaling Technology Europe, B.V.), Erk1/2 (cat. no. 9102; 1:1,000; Cell Signaling Technology Europe, B.V.) and GAPDH (cat. no. sc-25778; 1:1,000; Santa Cruz Biotechnology, Inc.). Subsequently, membranes were incubated with anti-rabbit or anti-mouse horseradish peroxidase conjugated IgG (cat. no. 111-035-003 and 115-035-003; 1:20,000; Jackson ImmunoResearch Laboratories, Inc.) for $1 \mathrm{~h}$ at room temperature. Bands were visualized by ECL-Plus detection reagents (Santa Cruz Biotechnology, Inc.).

Oncomine gene differential analysis by Oncomine. Differentially expressed HMMR mRNA between 586 ovarian serous cystadenocarcinoma and 8 normal ovarian tissues was analyzed by Oncomine (www.oncomine.org). Filters were set up as: Gene, HMMR, cancer type, ovarian cancer, dataset type, The Cancer Genome Atlas (TCGA) datasets. The data were grouped by cancer and normal type, and only samples included in the analysis are shown.

Statistical analysis. SPSS 20.2 software (IBM Corp.) was used to analyze the data. Data are presented as the mean \pm SEM. Differences between groups were assessed using a paired Student's t-test. $\mathrm{P}<0.05$ was considered to indicate a statistically significant difference. StarBase (version 2.0; http://starbase. sysu.edu.cn/) online database provided the correlation analysis between miR-411-5p expression and HMMR mRNA level. The correlation between miR-411-5p and HMMR was further investigated using patient sample information from the starBase online database.

\section{Results}

miR-411-5p is a potent suppressor of ovarian cancer proliferation, migration and invasion. The effects of miR-411-5p on the human ovarian cancer cell lines OVCAR-8 and SKOV3 were investigated using a miR-411-5p mimic. OVCAR-8 and SKOV3 cells were cultured and transfected with the negative control or the miR-411-5p mimic. At $48 \mathrm{~h}$ after transfection, the miR-411-5p expression level was significantly increased $(\mathrm{P}<0.001)$ in the miR-411-5p mimic groups, compared with the negative control groups, measured by RT-qPCR (Fig. 1). Furthermore, miR-411-5p significantly inhibited cell proliferation $(\mathrm{P}<0.001)$ in both the OVCAR-8 and the SKOV3 cell lines compared with the negative control, at all time points (Fig. 2A). Wound healing and Matrigel invasion assays were carried out to evaluate the effect of the

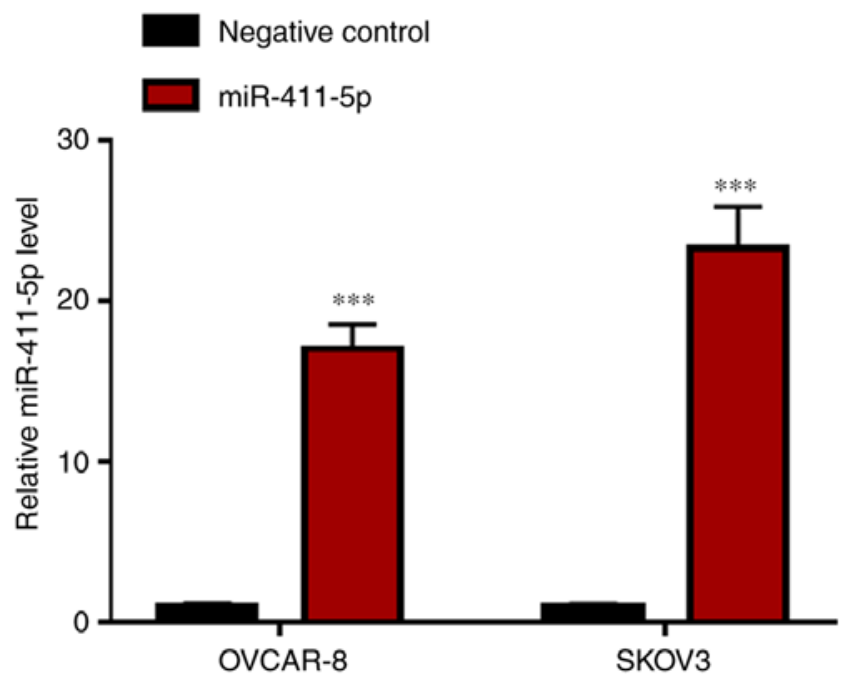

Figure 1. miR-411-5p mRNA levels in OVCAR-8 and SKOV3 cells following transfection with a negative control or miR-411-5p mimic, confirmed by reverse transcription-quantitative PCR. The Student's t-test was used to assess the difference. Error bars denote the SEM. ${ }^{* * *} \mathrm{P}<0.001$ vs. the negative control. miR, microRNA.

overexpression of miR-411-5p on the migration and invasion of ovarian cancer cells, respectively. The results of the cell wound healing assay suggested that wound healing was decreased $(\mathrm{P}<0.001)$ at $8 \mathrm{~h}(\mathrm{OVCAR}-8$ and SKOV3), $12 \mathrm{~h}$ (OVCAR-8 and SKOV3) and $16 \mathrm{~h}$ (SKOV3) in the miR-411-5p group compared with the negative control group (Fig. 2B), which indicated that miR-411-5p suppressed ovarian cancer cell migration. The results of the Matrigel invasion assay displayed that cell invasion was significantly suppressed $(\mathrm{P}<0.01)$ in the miR-411-5p group compared with the negative control group (Fig. 2C).

miR-411-5p directly targets HMMR. To identify the potential target of miR-411-5p in ovarian cancer cell lines, starBase (version 2.0; starbase.sysu.edu.cn) online database was used to predict the candidate downstream target genes. The results suggested that the 3'-UTR of HMMR contained putative binding sites for miR-411-5p (Fig. 3A). To further confirm whether HMMR was the direct target of miR-411-5p, the 3'-UTR of HMMR was synthesized and cloned into a luciferase reporter plasmid to construct dual luciferase reporter gene plasmids including the pmiR-HMMR 3'-UTR wild-type and pmiR-HMMR 3'-UTR mutant. The dual luciferase reporter gene plasmids were co-transfected into 293 cells with the negative control or miR-411-5p mimic. The results indicated that 293 cells co-transfected with the pmiR-HMMR 3'-UTR wild-type plasmid and the miR-411-5p mimic significantly decreased the relative luciferase activity compared with co-transfection with the negative control $(\mathrm{P}<0.001$; Fig. 3B). However, co-transfection with the pmiR vector or pmiR-HMMR 3'-UTR mutant plasmid plus the miR-411-5p or negative control mimic did not significantly influence the relative luciferase activity of 293 cells (Fig. 3B). These results illustrated that HMMR was a direct downstream target of miR-411-5p. The correlation between miR-411-5p and HMMR was further investigated using patient sample information from the starBase online database. miR-411-5p expression from miRNA sequencing displayed a significant negative correlation 
A
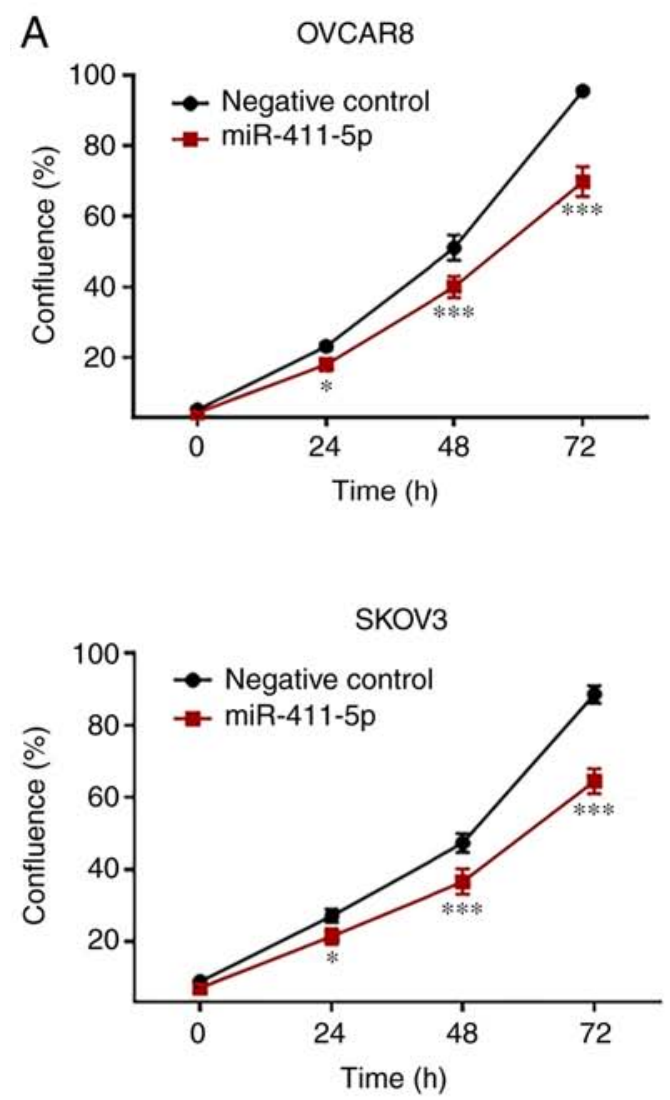

B

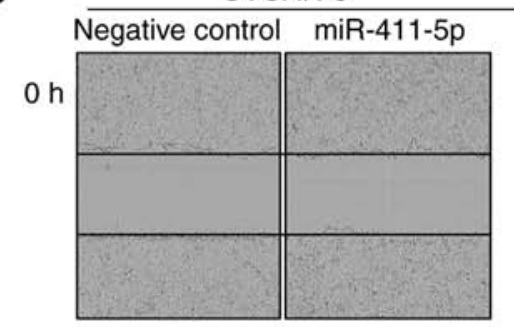

$12 \mathrm{~h}$
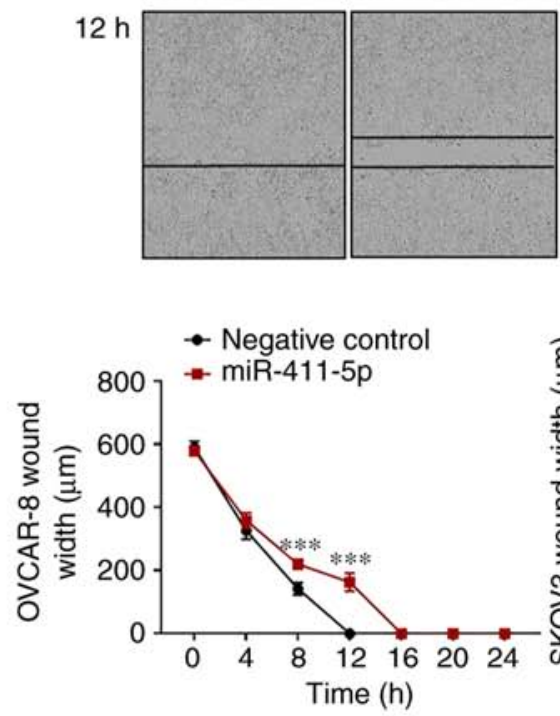

SKOV3
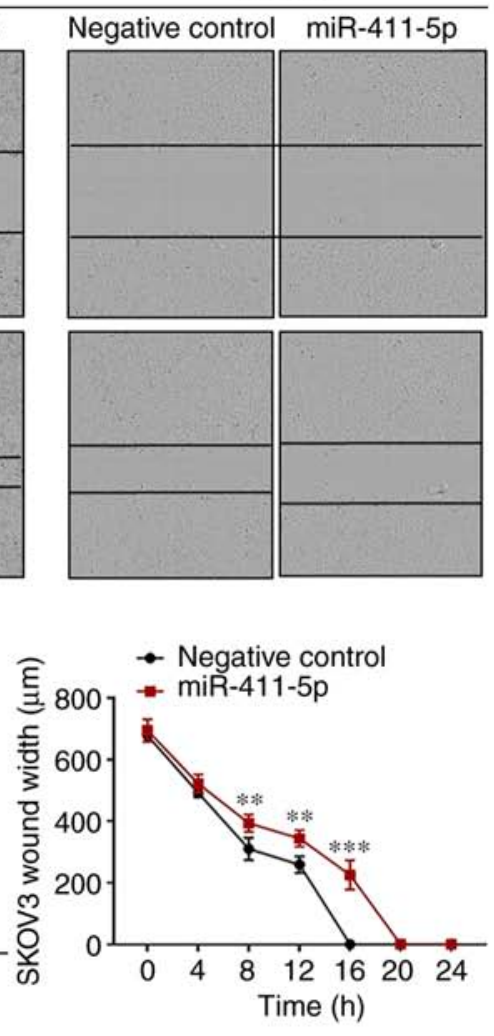

C
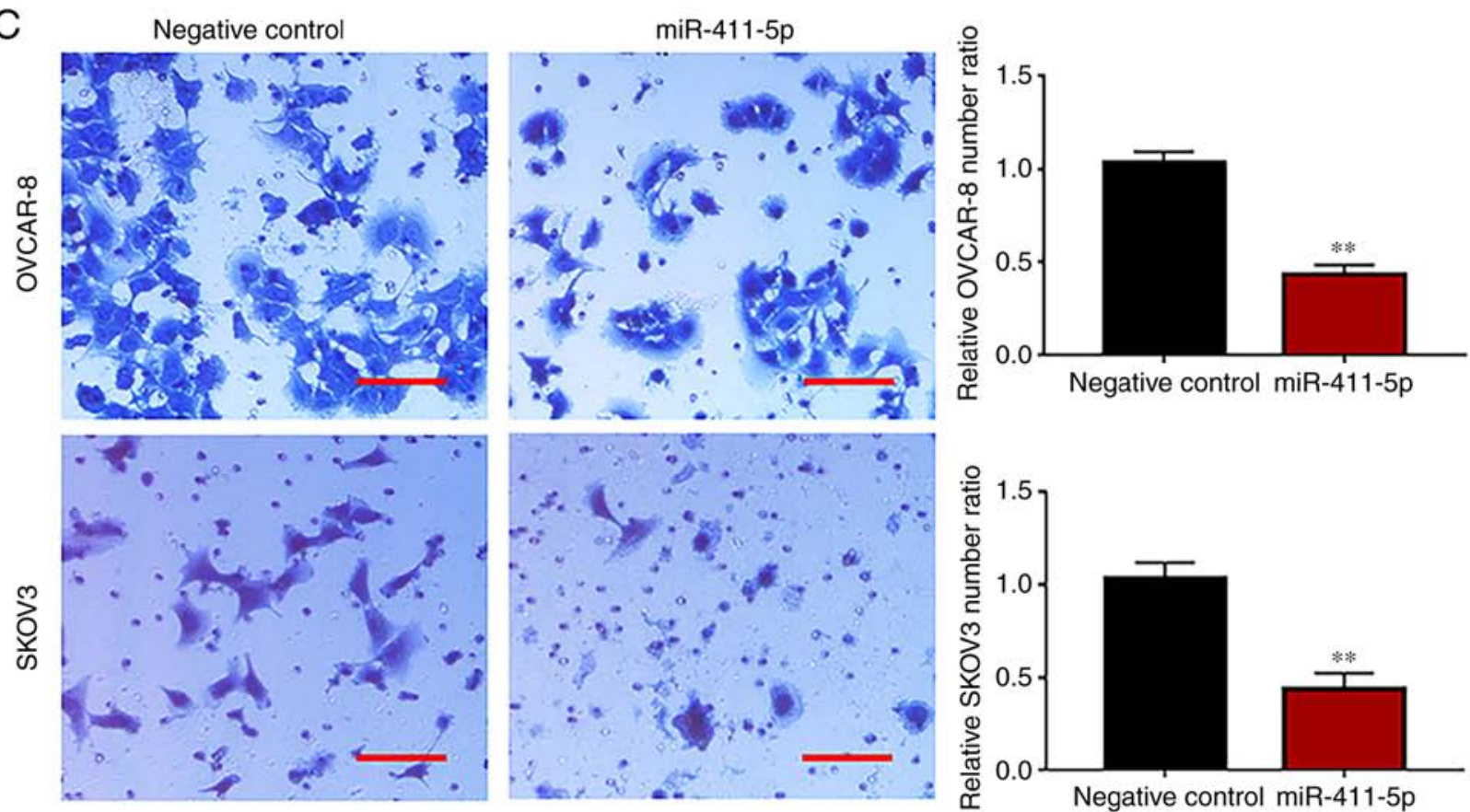

Figure 2. Cell behavior following transfection with the miR-411-5p mimic. (A) IncuCyte proliferation assay of OVCAR-8 and SKOV3 cells transfected with the negative control or miR-411-5p mimic. (B) Wound healing assay of OVCAR-8 and SKOV3 cells transfected with the negative control or miR-411-5p mimic (magnification, x10). (C) Matrigel invasion assay of OVCAR-8 and SKOV3 cells transfected with the negative control or miR-411-5p mimic. Scale bar length is $100 \mu \mathrm{m}$. The Student's t-test was used to assess the difference. Error bars denote the SEM. ${ }^{*} \mathrm{P}<0.05$ vs. the negative control; ${ }^{* *} \mathrm{P}<0.01 \mathrm{vs}$. the negative control; ${ }^{* * * *} \mathrm{P}<0.001$ vs. the negative control. miR, microRNA.

with HMMR mRNA level from RNA sequencing (correlation coefficient $r=-0.14809 ; \mathrm{P}=0.0158359$ ) from 265 ovarian serous cystadenocarcinoma patient samples (Fig. 3C) using the starBase Pan-Cancer miRNA-Target Expression Profile (24).
HMMR downregulation mediated by miR-411-5p overexpression may inhibit ovarian cancer cell proliferation by downregulating the activity of ERK1/2. The role of miR-411-5p and HMMR in the proliferation of ovarian cancer cells was 


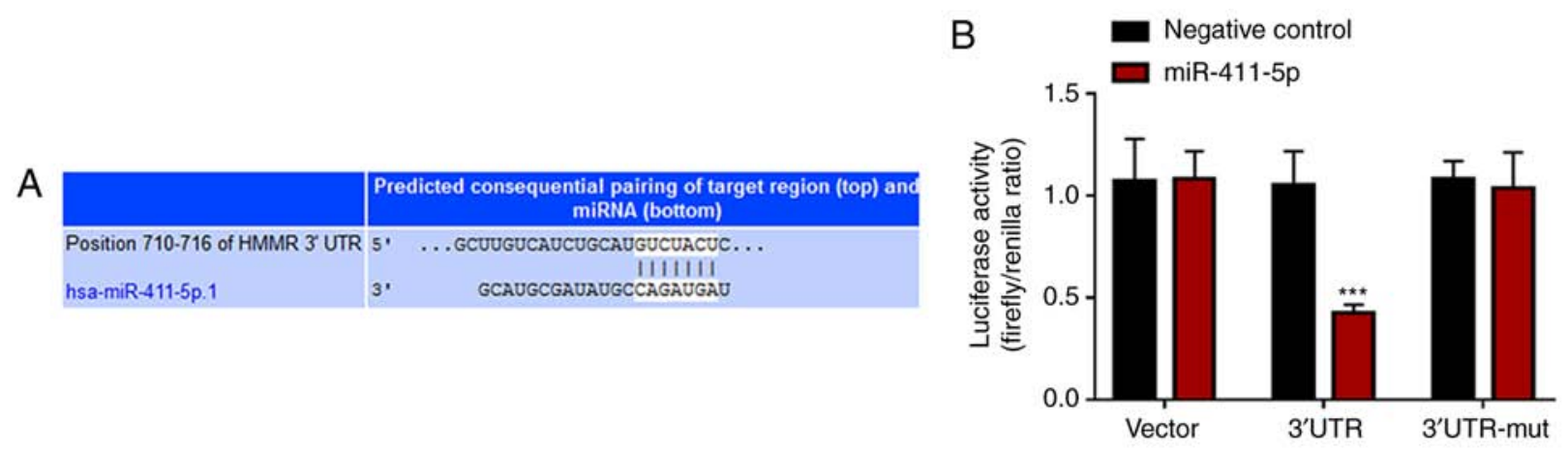

C

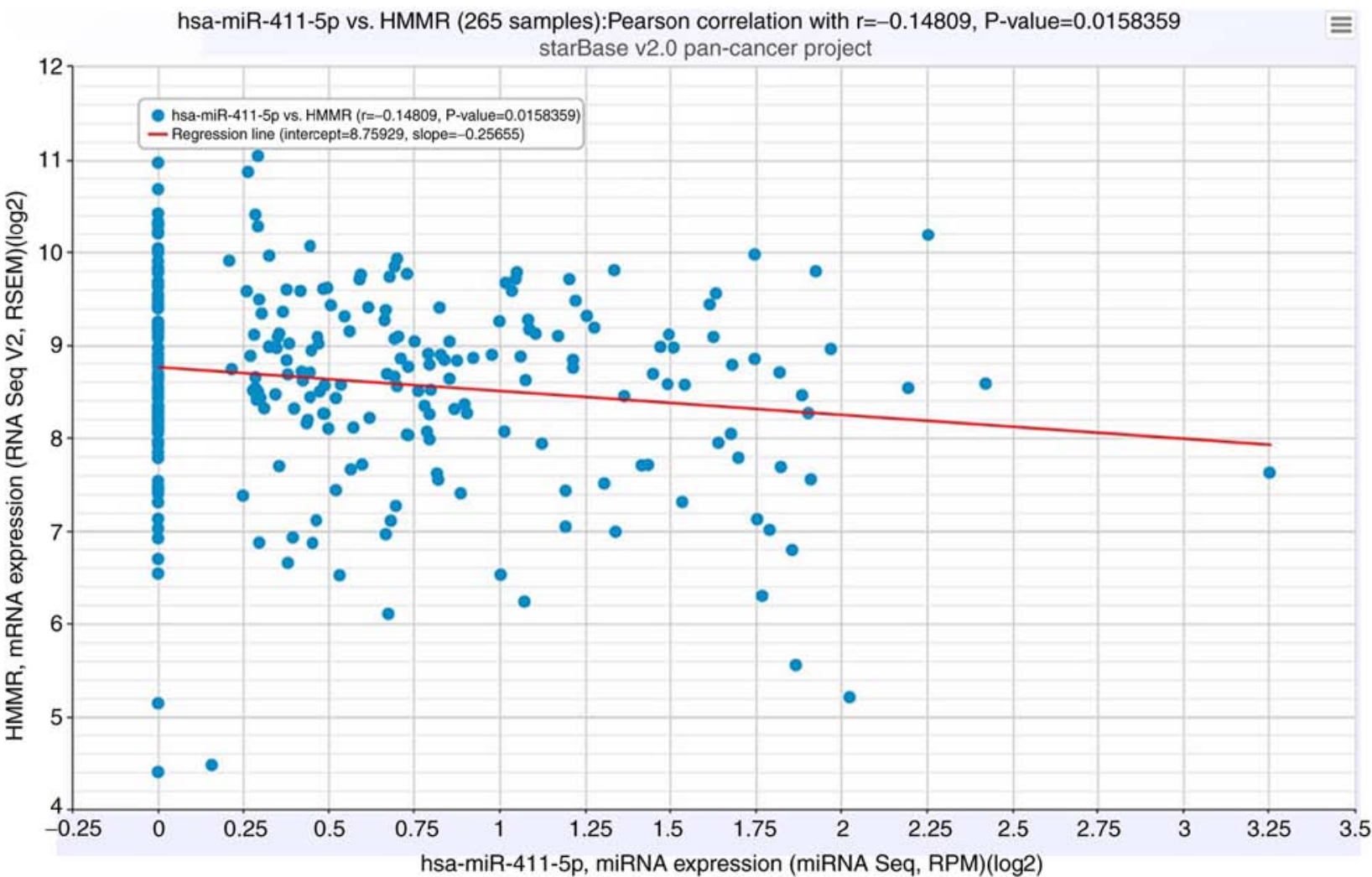

Figure 3. HMMR was identified as the direct downstream target of miR-411-5p in ovarian cancer cell lines. (A) StarBase (version 2.0; http://starbase.sysu.edu.cn/) online database predicted the 3'-UTR of HMMR contained putative binding sites of miR-411-5p. (B) Luciferase reporter assays revealed that miR-411-5p may bind to HMMR. The Student's t-test was used to assess the difference $(\mathrm{n}=3)$. Error bars denote the SEM. ${ }^{* * *} \mathrm{P}<0.001$ vs. negative control. (C) Correlation analysis between miR-411-5p expression and HMMR mRNA level. HMMR, hyaluronan mediated motility receptor; miR, microRNA; mut, mutant; RNA seq, RNA sequencing; $r$, correlation coefficient. RPM, reads/counts of exon model per million mapped reads; TPM (quantification of RSEM software), transcripts per kilobase of exonmodel per million mapped reads.

explored. The results suggested that ovarian cancer cells transfected with the miR-411-5p mimic displayed reduced HMMR mRNA and protein expression levels, as well as reduced ERK1/2 signaling pathway activity (Fig. 4A and B). Furthermore, differential HMMR mRNA expression between paired ovarian serous cystadenocarcinoma and normal tissue was analyzed by Oncomine. The results suggested that HMMR mRNA expression in ovarian serous cystadenocarcinoma was significantly higher $(\mathrm{P}<0.001)$ than that in normal ovarian tissue (Fig. 5).

\section{Discussion}

The biological role of miR-411 in ovarian cancer cells is not well understood (25), however, it has been reported that
miR-411 is downregulated in ovarian cancer cells (25). To the best of our knowledge, the present study is the first to demonstrate the regulatory molecular mechanism of miR-411-5p in the malignant biological behavior of ovarian cancer cells.

A number of previous studies have reported that miR-411 plays an important role as an oncogene in tumorigenesis by negatively regulating protein expression $(10,26)$. Further studies have reported that miR-411 may function as an oncogene in osteosarcoma, lung and hepatocellular carcinoma tumorigenesis and tumor development $(9,11,12,26,27)$. miR-411 promoted osteosarcoma cell proliferation and migration by inhibiting the expression of the metastasis suppressor protein 1 (MTSS1) (9). Furthermore, high miR-411 expression was an independent poor prognostic indicator for patients with non-small cell lung carcinoma, and compared with healthy 

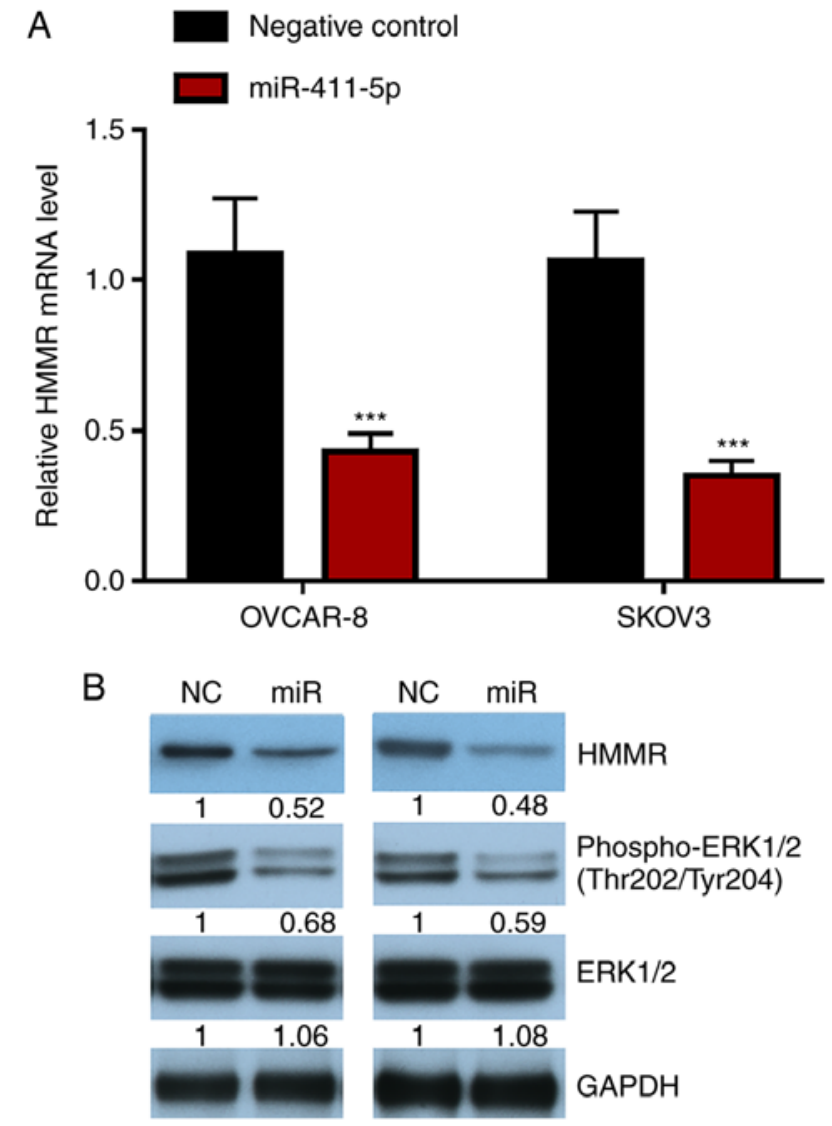

Figure 4. Molecular mechanisms of miR-411-5p in ovarian cancer cells. (A) Reverse transcription-quantitative PCR of HMMR mRNA levels in OVCAR-8 and SKOV3 cells $24 \mathrm{~h}$ post-transfection with the miR-411-5p mimic. (B) Western blot analysis of HMMR, phospho-ERK1/2, total ERK1/2 and GAPDH expression in OVCAR-8 and SKOV3 cells $72 \mathrm{~h}$ post-transfection with the miR-411-5p mimic. The Student's t-test was used to assess the difference $(\mathrm{n}=3)$. Error bars denote the SEM. ${ }^{* * *} \mathrm{P}<0.001$ vs. negative control. miR, microRNA; HMMR, hyaluronan mediated motility receptor; phospho, phosphorylated; NC, negative control.

controls, miR-411 expression levels were higher in the serum of patients with non-small cell lung carcinoma (26). Additionally, other studies have demonstrated that the oncogenic effect of miR-411 on lung cancer cell proliferation was mediated by direct downregulation of forkhead box O1 (10). miR-411 also increased cell migration in lung adenocarcinoma cells, was upregulated in tumors from patients who relapsed systemically and was associated with poor survival time following lung adenocarcinoma resection (11). Another study indicated that miR-411 promotes lung carcinogenesis by directly targeting the tumor suppressor genes sprouty RTK signaling antagonist 4 (SPRY4) and thioredocin interacting protein (27). Furthermore, it has been reported that miR-411 promoted hepatocellular carcinoma cell proliferation by targeting itchy E3 ubiquitin protein ligase (ITCH) (12).

Alternatively, numerous studies have reported that miR-411 functions as a tumor suppressor in oral, cervical, breast and colorectal cancer, as well as in renal cell carcinoma and glioma $(13,14,17,19,20)$. Recent study has shown that miR-411-5p was downregulated in oral cancer compared with non-cancerous tissue (13). Similarly, miR-411 was significantly downregulated and played a role as a tumor suppressor in renal cell carcinoma (14). In cervical cancer, miR-411 acted as a potential tumor suppressor to inhibit cancer progression by directly targeting STAT3 (15). Additionally, miR-411-5p inhibited proliferation and metastasis of breast cancer cells by directly targeting growth factor receptor bound protein 2 (GRB2) (17). Zhao et al (18) reported that miRNA-411 inhibited malignant biological behaviours of colorectal cancer cells by targeting phosphoinositide-3-kinase regulatory subunit 3 (PIK3R3). Additionally, miR-411-5p decreased glioma cell proliferation by targeting topoisomerase II- $\alpha$ (TOPO2A) (19); overexpression of $\mathrm{miR}-411-5 \mathrm{p}$ inhibited the proliferation of rhabdomyosarcoma cells in vitro and tumorigenicity in vivo (20); and miR-411-5p activated $\mathrm{p} 38$-mitogen activated protein kinase phosphorylation by directly downregulating SPRY4 and promoted apoptosis and myogenic differentiation (20). Furthermore, the miR-379/411 cluster regulated IL-18, significantly suppressed the invasive capacity of MESO1 cells (a tumor model) and contributed to drug resistance in malignant pleural mesothelioma (21).

A number of targets of miR-411 have been reported previously, including MTSS1 (9), TOP2A (19), ITCH (12), STAT3 (15), specificity protein 1 (16), GRB2 (17), PIK3R3 (18), SPRY4 (20), IL-18 (21), hypoxia inducible factor 1- $\alpha$ (28) and matrix metalloprotease-13 (29). In the present study, HMMR was identified as a novel direct target of miR-411-5p by a luciferase reporter assay. HMMR is required for bipolar spindle assembly and mitotic progression by integrating biochemical and structural pathways (30). HMMR expression is associated with poor prognosis and metastasis in non-small cell lung carcinoma (31). Furthermore, upregulation of HMMR in lung adenocarcinoma was associated with an inflammatory molecular signature and poor prognosis (32). Cell surface HMMR is an hyaluronic acid-binding protein that is not highly expressed in normal tissues but is commonly upregulated in a number of advanced forms of cancer $(33,34)$. However, the underlying mechanism of HMMR in ovarian cancer is not completely understood. Using TCGA, the present study suggested that HMMR mRNA expression is higher in ovarian serous cystadenocarcinoma than in normal ovarian tissue.

ERK1/2 kinases are ubiquitous and homologous mitogen activated protein kinases that mediate ligand-stimulated signals for the induction of proliferation, differentiation, survival, apoptosis, angiogenesis and motility (35). Upregulation and elevated activation of ERK1/2 kinases is common in human tumors (36-38). Indeed, studies have reported that in a large number of different types of cancer the ERK1/2 signaling pathway is constitutively active and may be involved in the pathogenesis of these tumors (36-38). Of note, the ERK signaling pathway plays a critical role in ovarian cancer pathogenesis and downregulation of this signaling pathway is highly effective for the inhibition of ovarian tumor proliferation (39). Furthermore, the nuclear localization of phosphorylated ERK1/2 can serve as a marker for the progression of ovarian cancer $(39,40)$.

HMMR is a known modulator of ERK1/2 in certain types of cancer $(41,42)$. HMMR overexpression resulted in activation of the ERK1/2 signaling pathway, which promotes cell proliferation of osteoblastic cells (41). Cell surface HMMR and CD44 act together and coordinate to sustain ERK1/2 signaling, leading to high basal motility of invasive breast 


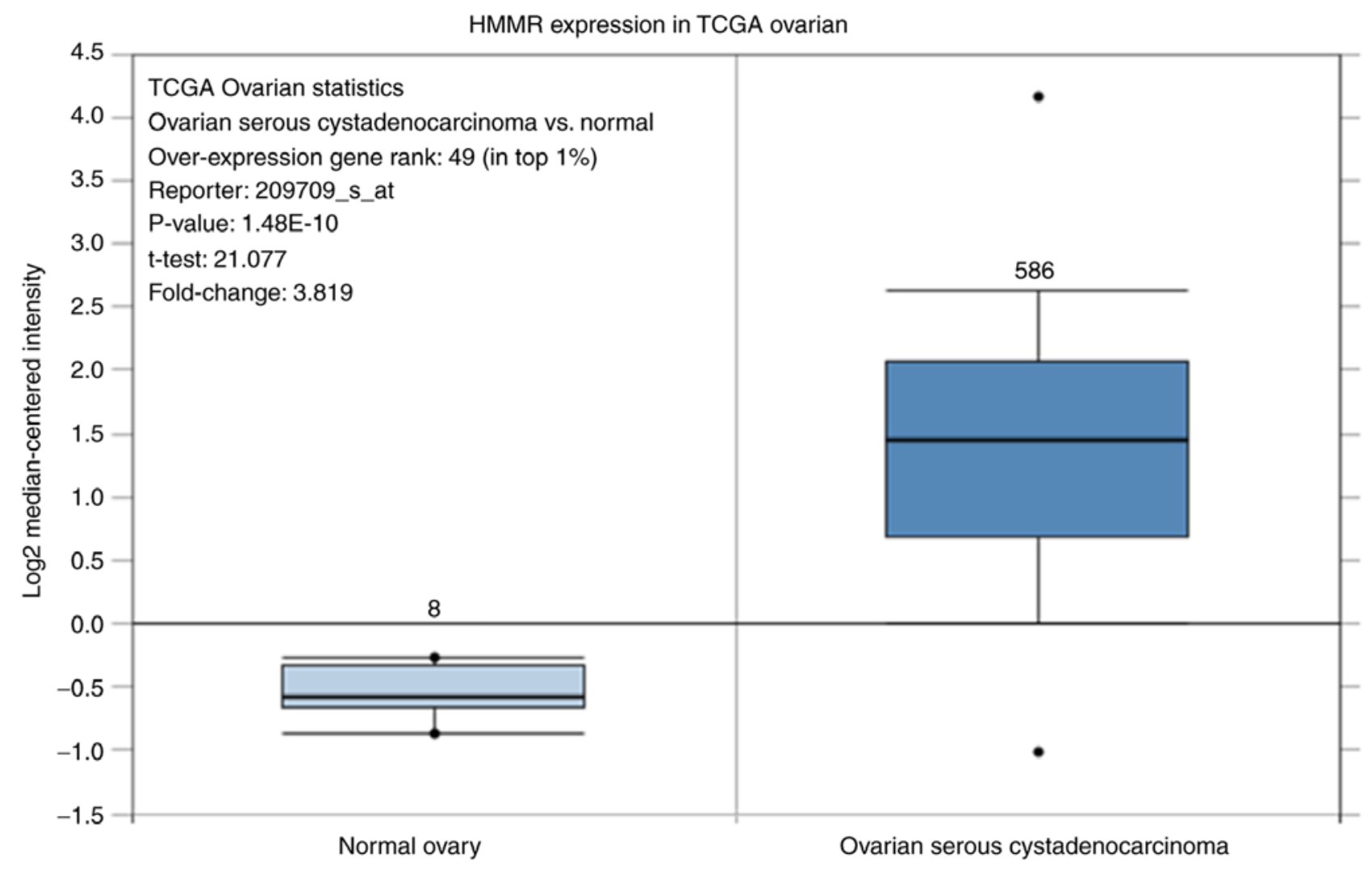

Figure 5. HMMR mRNA differential analysis of paired ovarian serous cystadenocarcinoma and normal tissues. HMMR, hyaluronan mediated motility receptor; TCGA, The Cancer Genome Atlas.

cancer cells (42). The results of the present study suggested that the expression of phosphorylated ERK1/2 and HMMR is decreased following the overexpression of $\mathrm{miR}-411-5 \mathrm{p}$ in ovarian cancer cells, a result that is consistent with the aforementioned studies. The present study suggested a potential interplay between HMMR and ERK1/2, via phosphorylated ERK1/2, which may contribute to cancer cell proliferation and motility modulation.

The present study indicated that the overexpression of miR-411-5p could suppress ovarian cancer cell (OVCAR-8 and SKOV3) proliferation and motility. In the present study, it was also described that miR-411-5p could downregulate HMMR, which resulted in the downregulation of the ERK1/2 signaling pathway activity, and thus inhibited ovarian cancer cell proliferation and motility. In different cancer types, miR-411-5p may have different roles $(12,19)$. In conclusion, the results of the present study demonstrated that miR-411-5p may be potential therapeutic target for ovarian cancer.

One limitation of the present study was that the results were based on miRNA mimic-mediated overexpression experiments in two ovarian cancer cell lines, where the miRNA level may be much higher than the endogenous miRNA level. Additionally, the wound healing should ideally be performed in $0 \% \mathrm{FBS}$, therefore the use of $4 \%$ FBS is a minor limitation. Future investigations, in particular in vivo experiments, should be performed to confirm the functional mechanisms and investigate the clinical application of miR-411-5p. Collectively, the present study provided a rationale for further investigation into miR-411-5p-based diagnosis, prognosis and targeted therapeutic strategies.

\section{Acknowledgements}

Not applicable.

\section{Funding}

The present study was funded by the Liaoning Province Science and Technology Foundation (grant no. 2019-ZD-0984) and the Shenyang Science and Technology Grant (grant no. 17-230-9-41).

\section{Availability of data and materials}

The datasets used and/or analyzed during the current study are available from the corresponding author on reasonable request.

\section{Authors' contributions}

FH, DZ and XN conceived and designed the study and drafted the manuscript. FH, DZ, CL, JN and XN performed the experiments. All authors analyzed and interpreted the data. All authors read and approved the final manuscript.

\section{Ethics approval and consent to participate}

Not applicable.

\section{Patient consent for publication}

Not applicable. 


\section{Competing interests}

The authors declare that they have no competing interests.

\section{References}

1. Torre LA, Bray F, Siegel RL, Ferlay J, Lortet-Tieulent J and Jemal A: Global cancer statistics, 2012. CA Cancer J Clin 65: 87-108, 2015

2. Siegel RL, Miller KD and Jemal A: Cancer Statistics, 2017. CA Cancer J Clin 67: 7-30, 2017.

3. Suh DH, Kim M, Kim K, Kim HJ, Lee KH and Kim JW: Major clinical research advances in gynecologic cancer in 2016: 10-year special edition. J Gynecol Oncol 28: e45, 2017.

4. Miller KD, Siegel RL, Lin CC, Mariotto AB, Kramer JL, Rowland JH, Stein KD, Alteri R and Jemal A: Cancer treatment and survivorship statistics, 2016. CA Cancer J Clin 66: 271-289, 2016.

5. Chornokur G, Amankwah EK, Schildkraut JM and Phelan CM: Global ovarian cancer health disparities. Gynecol Oncol 129: 258-264, 2013.

6. Croce CM: Causes and consequences of microRNA dysregulation in cancer. Nat Rev Genet 10: 704-714, 2009.

7. Zaman MS, Maher DM, Khan S, Jaggi M and Chauhan SC: Current status and implications of microRNAs in ovarian cancer diagnosis and therapy. J Ovarian Res 5: 44, 2012.

8. Langhe R: microRNA and ovarian cancer. Adv Exp Med Biol 889: 119-151, 2015

9. Xu N, Yang W, Liu Y, Yan F and Yu Z: MicroRNA-411 promoted the osteosarcoma progression by suppressing MTSS1 expression. Environ Sci Pollut Res Int 25: 12064-12071, 2018.

10. Zhao Z, Qin L and Li S: miR-411 contributes the cell proliferation of lung cancer by targeting FOXO1. Tumour Biol 37: 5551-5560, 2016.

11. Nadal E, Zhong J, Lin J, Reddy RM, Ramnath N, Orringer MB, Chang AC, Beer DG and Chen G: A MicroRNA cluster at 14q32 drives aggressive lung adenocarcinoma. Clin Cancer Res 20: 3107-3117, 2014

12. Xia K, Zhang Y, Cao S, Wu Y, Guo W, Yuan W and Zhang S: miR-411 regulated ITCH expression and promoted cell proliferation in human hepatocellular carcinoma cells. Biomed Pharmacother 70: 158-163, 2015

13. Zeljic K, Jovanovic I, Jovanovic J, Magic Z, Stankovic A and Supic G: MicroRNA meta-signature of oral cancer: Evidence from a meta-analysis. Ups J Med Sci 123: 43-49, 2018.

14. Zhang X, Zhang M, Cheng J, Lv Z, Wang F and Cai Z: MiR-411 functions as a tumor suppressor in renal cell cancer. Int J Biol Markers 32: e454-e460, 2017.

15. Shan D, Shang Y and Hu T: MicroRNA-411 inhibits cervical cancer progression by directly targeting STAT3. Oncol Res 27 349-358, 2018.

16. Guo L, Yuan J, Xie N, Wu H, Chen W, Song S and Wang X: miRNA-411 acts as a potential tumor suppressor miRNA via the downregulation of specificity protein 1 in breast cancer. Mol Med Rep 14: 2975-2982, 2016.

17. Zhang Y, Xu G, Liu G, Ye Y, Zhang C, Fan C, Wang H, Cai H, Xiao R, Huang Z and Luo Q: miR-411-5p inhibits proliferation and metastasis of breast cancer cell via targeting GRB2. Biochem Biophys Res Commun 476: 607-613, 2016.

18. Zhao J, Xu J and Zhang R: MicroRNA-411 inhibits malignant biological behaviours of colorectal cancer cells by directly targeting PIK3R3. Oncol Rep 39: 633-642, 2018.

19. Deguchi S, Katsushima K, Hatanaka A, Shinjo K, Ohka F, Wakabayashi T, Zong H, Natsume A and Kondo Y: Oncogenic effects of evolutionarily conserved noncoding RNA ECONEXIN on gliomagenesis. Oncogene 36: 4629-4640, 2017.

20. Sun M, Huang F, Yu D, Zhang Y, Xu H, Zhang L, Li L, Dong L, Guo L and Wang S: Autoregulatory loop between TGF- $\beta 1 / \mathrm{miR}-411-5 \mathrm{p} / \mathrm{SPRY} 4$ and MAPK pathway in rhabdomyosarcoma modulates proliferation and differentiation. Cell Death Dis 6: e1859, 2015.

21. Yamamoto K, Seike M, Takeuchi S, Soeno C, Miyanaga A, Noro R, Minegishi Y, Kubota K and Gemma A: MiR-379/411 cluster regulates IL-18 and contributes to drug resistance in malignant pleural mesothelioma. Oncol Rep 32: 2365-2372, 2014.

22. Kozomara A, Birgaoanu M and Griffiths-Jones S: miRBase: From microRNA sequences to function. Nucleic Acids Res 47: D155-D162, 2019.
23. Livak KJ and Schmittgen TD: Analysis of relative gene expression data using real-time quantitative PCR and the 2(-Delta Delta C(T)) method. Methods 25: 402-408, 2001.

24. Yang JH, Li JH, Shao P, Zhou H, Chen YQ and Qu LH: starBase: A database for exploring microRNA-mRNA interaction maps from Argonaute CLIP-Seq and Degradome-Seq data. Nucleic Acids Res 39: D202-D209, 2011.

25. Kim YW, Kim EY, Jeon D, Liu JL, Kim HS, Choi JW and Ahn WS: Differential microRNA expression signatures and cell type-specific association with Taxol resistance in ovarian cancer cells. Drug Des Devel Ther 8: 293-314, 2014

26. Wang SY, Li Y, Jiang YS and Li RZ: Investigation of serum miR-411 as a diagnosis and prognosis biomarker for non-small cell lung cancer. Eur Rev Med Pharmacol Sci 21: 4092-4097, 2017.

27. Zhang C, Wang H, Liu X, Hu Y, Ding L, Zhang X, Sun Q and Li Y: Oncogenic microRNA-411 promotes lung carcinogenesis by directly targeting suppressor genes SPRY4 and TXNIP. Oncogene 38: 1892-1904, 2019.

28. Ai P, Shen B, Pan H, Chen K, Zheng J and Liu F: MiR-411 suppressed vein wall fibrosis by downregulating MMP-2 via targeting HIF-1 $\alpha$. J Thromb Thrombolysis 45: 264-273, 2018.

29. Wang G, Zhang Y, Zhao X, Meng C, Ma L and Kong Y: MicroRNA-411 inhibited matrix metalloproteinase 13 expression in human chondrocytes. Am J Transl Res 7: 2000-2006, 2015.

30. Chen H, Mohan P, Jiang J, Nemirovsky O, He D, Fleisch MC, Niederacher D, Pilarski LM, Lim CJ and Maxwell CA: Spatial regulation of Aurora A activity during mitotic spindle assembly requires RHAMM to correctly localize TPX2. Cell Cycle 13: 2248-2261, 2014

31. Wang D, Narula N, Azzopardi S, Smith RS, Nasar A, Altorki NK, Mittal V, Somwar R, Stiles BM and Du YN: Expression of the receptor for hyaluronic acid mediated motility (RHAMM) is associated with poor prognosis and metastasis in non-small cell lung carcinoma. Oncotarget 7: 39957-39969, 2016.

32. Stevens LE, Cheung WKC, Adua SJ, Arnal-Estapé A, Zhao M, Liu Z, Brewer K, Herbst RS and Nguyen DX: Extracellular matrix receptor expression in subtypes of lung adenocarcinoma potentiates outgrowth of micrometastases. Cancer Res 77: 1905-1917, 2017.

33. Turley EA, Noble PW and Bourguignon LY: Signaling properties of hyaluronan receptors. J Biol Chem 277: 4589-4592, 2002.

34. Adamia S, Maxwell CA and Pilarski LM: Hyaluronan and hyaluronan synthases: Potential therapeutic targets in cancer. Curr Drug Targets Cardiovasc Haematol Disord 5: 3-14, 2005.

35. Roskoski R Jr: ERK1/2 MAP kinases: Structure, function, and regulation. Pharmacol Res 66: 105-143, 2012

36. Holmström TH, Tran SE, Johnson VL, Ahn NG, Chow SC and Eriksson JE: Inhibition of mitogen-activated kinase signaling sensitizes HeLa cells to Fas receptor-mediated apoptosis. Mol Cell Biol 19: 5991-6002, 1999.

37. Huang C, Jacobson $\mathrm{K}$ and Schaller MD: MAP kinases and cell migration. J Cell Sci 117: 4619-4628, 2004

38. Mansour SJ, Resing KA, Candi JM, Hermann AS, Gloor JW, Herskind KR, Wartmann M, Davis RJ and Ahn NG: Mitogen-activated protein (MAP) kinase phosphorylation of MAP kinase kinase: Determination of phosphorylation sites by mass spectrometry and site-directed mutagenesis. J Biochem 116: 304-314, 1994.

39. Steinmetz R, Wagoner HA, Zeng P, Hammond JR, Hannon TS, Meyers JL and Pescovitz OH: Mechanisms regulating the constitutive activation of the extracellular signal-regulated kinase (ERK) signaling pathway in ovarian cancer and the effect of ribonucleic acid interference for ERK1/2 on cancer cell proliferation. Mol Endocrinol 18: 2570-2582, 2004.

40. Amsterdam A, Shezen E, Raanan C, Schreiber L, Prus D, Slilat Y, Ben-Arie A and Seger R: Nuclear localization of phosphorylated ERK1 and ERK2 as markers for the progression of ovarian cancer. Int J Oncol 39: 649-656, 2011.

41. Hatano H, Shigeishi H, Kudo Y, Higashikawa K, Tobiume K, Takata $\mathrm{T}$ and Kamata N: Overexpression of receptor for hyaluronan-mediated motility (RHAMM) in MC3T3-E1 cells induces proliferation and differentiation through phosphorylation of ERK1/2. J Bone Miner Metab 30: 293-303, 2012.

42. Hamilton SR, Fard SF, Paiwand FF, Tolg C, Veiseh M, Wang C, McCarthy JB, Bissell MJ, Koropatnick J and Turley EA: The hyaluronan receptors CD44 and Rhamm (CD168) form complexes with ERK1,2 that sustain high basal motility in breast cancer cells. J Biol Chem 282: 16667-16680, 2007.

This work is licensed under a Creative Commons Attribution-NonCommercial-NoDerivatives 4.0 International (CC BY-NC-ND 4.0) License. 